\title{
BMJ Open Diagnostic accuracy of shear wave elastography for prediction of breast malignancy in patients with pathological nipple discharge
}

Xiaobo Guo, ${ }^{1}$ Ying Liu, ${ }^{1}$ Wanhu $\mathrm{Li}^{2}$

To cite: Guo X, Liu Y, Li W. Diagnostic accuracy of shear wave elastography for prediction of breast malignancy in patients with pathological nipple discharge. BMJ Open 2016;6:e008848. doi:10.1136/bmjopen-2015008848

- Prepublication history for this paper is available online. To view these files please visit the journal online (http://dx.doi.org/10.1136/ bmjopen-2015-008848).

Received 21 May 2015 Revised 13 October 2015 Accepted 2 November 2015

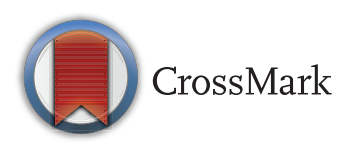

\begin{abstract}
${ }^{1}$ Department of Ultrasonography, 476 Clinical Department, Fuzhou General Hospital of Nanjing Military Region, Fuzhou, China 2Department of Radiology, Shandong Cancer Hospital and Institute, Jinan, China
\end{abstract}

Correspondence to Dr Xiaobo Guo; 782605810@qq.com and Wanhu Li; Iwhvzg@126.com

\section{ABSTRACT}

Objectives: Pathological nipple discharge (PND) may indicate malignant breast lesions. As the role of shear wave elastography (SWE) in predicting these malignant lesions has not yet been evaluated, we aim to evaluate the diagnostic value of SWE for this condition.

Design: Prospective diagnostic accuracy study comparing a combination of qualitative and quantitative measurements of SWE (index test) to a ductoscopy and microdochectomy for histological diagnosis (reference test).

Setting: Fuzhou General Hospital of Nanjing military command.

Participants: A total of 379 patients with PND were finally included from January, 2011 to March 2014, after we screened 1084 possible candidates. All participants were evaluated through SWE, with qualitative parameters generated by Virtual Touch tissue imaging (VTI) and quantitative parameters generated by Virtual Touch tissue quantification (VTQ). All the patients were consented to receive a ductoscopy and microdochectomy for histological diagnosis, and the results were set as a reference test.

Outcome measures: Sensitivity and specificity of the combined VTI and VTQ of the SWE for detection of malignancy in patients with PND.

Results: The 379 participants presented with 404 lesions. The results of pathological examination showed that $326(80.7 \%)$ of the 404 lesions were benign and the other $78(19.3 \%)$ were malignant. An area under the curve of elasticity score, VTQm and VTQc, were $0.872,0.825$ and 0.857 , respectively, with the corresponding cut-off point as $2.50,2.860 \mathrm{~m} / \mathrm{s}$ and $3.015 \mathrm{~m} / \mathrm{s}$, respectively. After a combination of these measurements, the sensitivity, specificity, and positive and negative predictive value (PPV and NPV), were $89.7 \%, 72.1 \%, 43.5 \%$ and $96.7 \%$, respectively. The sensitivity analysis showed $82 \%$ of the sensitivity and $96.8 \%$ of the specificity, in which patients with no pathological findings in ductoscopy were excluded. Conclusions: Ultrasonographic elastography is sensitive for patients with PND and could be used as a triage test before ductoscopy examination. Studies for further improvement of diagnostic sensitivity are warranted.

\section{Strengths and limitations of this study}

- Diagnostic accuracy of shear wave elastography (SWE) for detecting malignancy of patients with PND has rarely been studied.

- For the first time, this study tested diagnostic accuracy of a synthesised measurement of qualitative and quantitative measures of SWE for detecting malignancy in patients with PND.

- Limitations include the fact that the weight of each measurement in the synthesised score was assigned evenly and the surgeon was not blinded.

\section{INTRODUCTION}

In 2014, the American Cancer Society reported 235030 new cases of breast cancer and 40430 breast cancer-related deaths. ${ }^{1}$ The prevalence rate of breast cancer is the highest of all types of malignant carcinoma in Chinese women. ${ }^{2}$ The cases of Chinese women with breast cancer account for $12.2 \%$ of all newly diagnosed breast cancer around the world and $9.6 \%$ of deaths from breast cancer. $^{2}$

Pathological nipple discharge (PND) is the third most common symptom of patients, prompting referral to doctors for breast diseases. ${ }^{3}$ PND is believed to be an indicator for breast carcinoma, especially when the nipple discharge is bloody. ${ }^{3}$ Mastectomies were unnecessarily carried out without obtaining a histopathological diagnosis before the year 1950. Although mastectomies are not necessarily needed nowadays, patients with PND would normally be advised to undergo further examinations to rule out breast malignancy. These examinations involve mammography, ultrasonography, ductoscopy with or without galactography, as well as cytological tests. Mammography and B-mode ultrasonography (BUS) are used for screening patients with high risk of breast 
carcinoma, but they are not sensitive enough for detecting malignancy in patients with PND, since small lesions may be occult with these two modalities. ${ }^{4}$ Ductoscopy and microdochectomy are the gold standard for diagnosis of malignancy in patients with PND. ${ }^{5}{ }^{6}$ In clinical practice, patients with PND are conventionally advised a ductoscopy, but ductoscopy and microdochectomy are invasive examinations with high cost, so some patients refuse ductoscopy and turn to other non-invasive tests instead. Cytological tests can only be used as supportive evidence. $^{7}$ Therefore, a triage test is needed before patients with PND are asked to take a ductoscopy and microdochectomy examination.

Ultrasonographic elastography works through the assumption that tissue compression produces strain within the tissue, and that the strain is less in firm tissue than in soft tissue. So the firmness of the tissue to predict malignancy can be evaluated through measuring the tissue strain ratio, which is usually measured as elasticity. ${ }^{8}$ Recently, several studies indicated that ultrasonographic elastography may be a useful tool for differentiating benign and malignant masses. The sensitivity of predicting malignancy in patients with breast lesions ranges from $80 \%$ to $98 \%$, while the specificity ranges from $66 \%$ to $84 \% .{ }^{9-14}$ Recently, a shear wave elastography (SWE) was reported to be less operator-dependent, and predictive of ductal or intraductal carcinoma. ${ }^{8}{ }^{15} 16$ A five-level elasticity score is normally used for qualitative assessment of the images of elastography, while a quantitative measurement of elastography is also becoming popular; a combination of these ultrasonic measurements seems to be beneficial for improving diagnostic accuracy of breast malignancies. ${ }^{816}$ We noticed that the diagnostic value of SWE to detect malignancy in patients with PND has rarely been studied, especially with a combination of qualitative and quantitative measurements of SWE. So we conducted a study aiming to find out the sensitivity and specificity accuracy of SWE for predicting malignancy in patients with PND.

\section{METHODS AND MATERIALS}

This study is designed for evaluating the diagnostic accuracy of SWE for detection of malignancy in patients with PND. The study protocol is approved by the ethical review board in Fuzhou General Hospital of Nanjing Military Command (number FZJQ2011018), where the study was conducted and the data were collected. Data collection was planned before the index and reference test.

\section{Patients}

Patients with PND symptoms less than a year in duration were recruited from outpatient settings in the Fuzhou General Hospital of Nanjing Military Command. PND was defined as nipple discharge in women who stopped breastfeeding and were not pregnant. ${ }^{17}$ The patients were screened for eligibility according to the following inclusion criteria: (1) an age over 18 years; (2) with or without palpable breast masses; (3) agreeing to participate in this study; (4) providing written consent for further examination, biopsy and use of the study data. We excluded participants who (1) refused to receive a ductoscopy and microdochectomy or a biopsy; (2) had serious damage of internal organs, which could potentially bias the results; (3) could not receive local anaesthesia because of allergy; (4) were taking or had taken chemotherapy or radiotherapy for malignant breast tumours.

\section{Ultrasonographic imaging}

Eligible participants were asked to take ultrasonographic examinations before undergoing ductoscopy and microdochectomy. The ultrasonographic examinations included a BUS and an elastography with acoustic radiation force impulse (ARFI), which were performed with a Siemens ACUSON S2000 ultrasonographic system (Siemens Ltd, China, product standard: YZB/USA 3876-2010). One operator (with 10 years of experience in ultrasonography imaging) ran the BUS and the ARFI to get the BUS and elastography images, using a superficial probe (9L4, Siemens Ltd, China) with a frequency of 4-9 Hz.

The BUS images of the breast lesions were obtained and categorised according to the Breast Imaging Reporting and Data System (BI-RADS). In the BI-RADS, the number 1 refers to negative findings; number 2 refers to benign findings; number 3 refers to probably benign findings; number 4 refers to suspicious malignancy; and number 5 is highly suggestive of malignancy. Moreover, we applied a subclassification scheme for BI-RADS 4: (1) 4A: low suspicious for malignancy; (2) 4B: intermediate suspicious for malignancy; (3) 4C: moderate concern, but not classic for malignancy. ${ }^{18}$ Two radiologists (XBG and YL, who each have at least 5 years of experience in ultrasonography) independently assessed the BUS images for the following aspects: the shape of the mass, its depth, orientation and margin, boundary of the lesions, echo pattern, posterior acoustic features as well as surrounding tissue changes. All the detected masses were divided into two types (solid and cystic) according to the presence of a cystic portion. In cases involving a solid mass in a dilated duct (intraductal) or cyst (intracystic) with a predominant cystic portion, the radiologists considered the cases as cystic type lesions.

After the BUS examination, the operator switched the ultrasonic mode to virtual touch quantification (VTQ). The patients were told to hold their breath ,while the operator detected the inside and margin of the lesion, with a previous set region of interest (ROI) in a fixed area of $5 \times 5 \mathrm{~cm}$. The operator recorded the value of VTQ $(\mathrm{m} / \mathrm{s})$ and the depth of the ROI $(\mathrm{cm})$ from skin for each measurement, in a total of five measurements. We calculated the mean value of the five measurements as the final VTQ score. Additionally, the value of VTQ was measured at both the margin (VTQm) and centre 
(VTQc) of the breast lesions. Measurable SWE ranges from 0 to $9 \mathrm{~m} / \mathrm{s}$, a value of ROI exceeding the range would be shown as 'X.XX $\mathrm{m} / \mathrm{s}$ ' on the screen. Participants with this ' $\mathrm{X} . \mathrm{XX} \mathrm{m} / \mathrm{s}$ ' value were excluded.

After finishing the examination using the VTQ mode, the operators switched to virtual touch imaging (VTI). ARFI sequences were generated to evaluate the elasticity scores of the breast lesions in VTI mode. The VTI images were evaluated and categorised through a five-level scoring system. ${ }^{19}$ An elasticity score of 1 indicates strain seen in the entire hypoechoic lesion (the entire lesion is equally shaded in green); score 2 indicates that strain is not seen in part of the hypoechoic lesion (the lesion is shown as a mosaic of green and blue); score 3 indicates that strain is seen in the peripheral areas only (peripheral areas in green); score 4 indicates no strain seen in the entire hypoechoic area (lesion is shown in blue); score 5 indicates no strain in the entire hypoechoic lesion or the surrounding area (both the entire hypoechoic lesion and its surrounding area are shown in blue). A higher elasticity score indicates stiffer tissue. For cystic-type lesions, we analysed the elasticity scores in solid areas. After the BUS and ARFI elastography examination, two radiologists blinded to the medical history of the participants independently reviewed the images and measurements. Any discrepancy was solved by discussion.

\section{Microdochectomy and pathological examination}

After finishing the examination of BUS imaging and elastography, the participants received a ductoscopy examination. Microdochectomy was performed to get a biopsy sample if the ductoscopy indicated a breast lesion. The result of microdochectomy and pathological examination was set as a reference test (the gold standard). The patients were asked to stay in supine position with the arm preferably extending on a sideboard. After skin disinfection, the fluid-producing ducts were identified by massaging the breast towards the nipple. After dilating the target ducts with lacrimal dilators, a surgeon (with 5 years of experience in microdochectomy) introduced an endoscope (a fiberoptic ductoscope, $0.8 \mathrm{~mm}$ of sheath, $80 \mathrm{~mm}$ of length; manufactured in Denzlingen, Germany) through the ducts. The surgeon was not blinded from the results of the ultrasonographic examinations. After inserting the ductoscopy, the surgeon injected saline into the ducts to keep them expanded. The ductoscopy was advanced through the ducts under direct vision from the endoscope camera; all tiers of branches were examined until the endoscope could not be advanced further or an obstructing lesion was detected. When an obstructing lesion was found, the image of this lesion would be taken using an endoscope camera, moreover, an endoscopy-guided biopsy would be performed as scheduled. After biopsy and image taking, the patient was referred to a surgeon for further treatment advice.
After detection of ductal lesions, methylene blue was injected into the discharged mammary duct. Local anaesthesia was performed and an incision around the areola implemented. The pathological duct was separated according to tissue with methylene-blue stain, and the duct was then dissected and excised. The operator then pulled the clip out of the duct together with the scope after the excision was finished. Biopsy samples over $1 \mathrm{~mm}^{3}$ were fixed using formalin after sample collection and were sent for histological examination.

\section{Statistical analysis}

Statistical analysis was performed to specify the diagnostic sensitivity and specificity of a synthesised measurement of VTI (elasticity score) and VTQ measurements. First, we would assess the interobserver variability between the two radiologists (XBG and YL) using the Cohen's $\kappa$ test. The value of $\kappa$ over 0.75 was considered as excellent, 0.40 0.75 as fair to good, and below 0.40 as poor. Second, we calculated the sensitivity, specificity and the area under the curve (AUC) of the elasticity score, VTQm and VTQc, using the receiver operating characteristics (ROC) curve. A 0.5 value of the AUC was considered as no diagnostic value; $0.5-0.7$, low diagnostic value; $0.7-0.9$, medium diagnostic value; 0.9-1.0, high diagnostic value. ${ }^{20}$ We calculated the cut-off point of the elasticity score, VTQm and VTQc, and gave the sensitivity and specificity, respectively, based on both the highest sum of sensitivity and specificity, and a relatively high sensitivity of the measurements. According to the cut-off points, we synthesised the measurements by the following formula: the synthesised score=elasticity score + VTQm + VTQc. A synthesised score of 1 was given if a value over the cut-off point was observed in a breast lesion; otherwise, a score of -1 was given. A breast lesion with a final score below 0 was classified as benign, otherwise it was categorised as malignant. Third, after differentiating benign and malignant lesions using the synthesised score, we evaluated the sensitivity, specificity, positive predictive value (PPV) and negative predictive value (NPV). The diagnostic value of this synthesised measurement was compared with the biopsy results, using the McNemar's test. We also compared the BI-RADS with the synthesised measurement using the $\chi^{2}$ test. All statistical analysis was performed using Statistical Package for the Social Sciences (SPSS, V.20.0, Armonk, IBM Corp, New York, USA) and R software (http://www.r-project.org, V.3.1.1).

\section{RESULTS}

A total of 379 patients with PND were included in this study, after 1084 possible candidates were screened and 705 of them excluded. The 379 participants presented with 404 lesions, while 25 participants showed breast lesions on both sides. The process of eligibility screening and a brief description of the results of the BUS, elastography, ductoscopy and microdochectomy examinations, are shown in figure 1. The age of the included 
participants ranged from 25 to 67 years. The results of the pathological examination showed that $326(80.7 \%)$ of the 404 lesions were benign and $78(19.3 \%)$ were malignant. Table 1 shows the types of benign and malignant lesions. Figures 2 and 3 show examples of invasive ductal carcinoma and fibroadenoma diagnosed with BUS and elastography. Of all the breast lesions, 191 (47.2\%) were palpable masses; 127 were benign and 64 were malignant. A total of 59 participants complained of bloody discharge in 62 breast lesions (15.3\%); 9 were benign and 53 were malignant. The size of the lesions ranged from $3.2 \mathrm{~mm}$ to $38.7 \mathrm{~mm}$. The benign lesions showed a mean size of $8.16 \mathrm{~mm}$, while the malignant lesions showed a mean size of $15.58 \mathrm{~mm}$. No patients with SWE exceeding the range were found. Moderate or severe pain (with a score of visual analogue scale over $50 \mathrm{~mm}$ ) in the breast was reported by 133 of the 227 participants who received ductoscopy. Other adverse events were not reported. The agreement between the two radiologists for ultrasound elastography was $\operatorname{good}(\kappa=0.76$, $\mathrm{p}<0.01)$.

The diagnostic cut-off points of the elasticity score, VTQm and VTQC

We calculated the cut-off points for elasticity scores, VTQm and VTQc. The results showed that a cut-off point of 2.50 for elasticity score yielded a sensitivity of 0.872 (95\% CI 0.795 to 0.940 ), a specificity of 0.736 (95\% CI 0.660 to 0.805 ) and an AUC of 0.854 . We also calculated a cut-off point of $2.860 \mathrm{~m} / \mathrm{s}$ for VTQm with a sensitivity of 0.731 (95\% CI 0.628 to 0.821 ), a specificity of 0.742 (95\% CI 0.673 to 0.805$)$ and an AUC of 0.825 . Additionally, a cut-off point of $3.015 \mathrm{~m} / \mathrm{s}$ was calculated
Table 1 Distribution of benign and malignant lesions classified by microdochectomy

\begin{tabular}{ll}
\hline Diagnosis after biopsy & Number of lesions (\%) \\
\hline Benign & 326 \\
No pathological lesions & $167(51.2)$ \\
Fibrocystic breast disease & $82(25.1)$ \\
Fibroadenoma & $60(18.4)$ \\
Atypical ductal hyperlasia & $11(3.5)$ \\
Intraductal pailloma & $6(1.8)$ \\
Malignant & 78 \\
Invasive ductal carcinoma & $61(78.2)$ \\
Ductal carcinoma in situ & $6(7.7)$ \\
Papillary carcinoma & $7(9.0)$ \\
Lobular carcinoma & $4(5.1)$ \\
\hline
\end{tabular}

for VTQc with a sensitivity of 0.731 (95\% CI 0.628 to 0.821 ), a specificity of 0.698 (95\% CI 0.616 to 0.761 ) and an AUC of 0.857 . Table 2 shows raw numbers and diagnostic estimates of the elasticity score, VTQm and VTQc. Figure 4 shows the ROC curve of each measurement.

\section{Diagnostic accuracy of the synthesised measurement} of the elasticity score, VTQm and VTQc

We used a synthesised measurement of both quantitative and qualitative parameters to predict malignant breast lesions. The measurement categorised 243 (60.1\%) breast lesions as benign and $161(39.9 \%)$ as malignant. Table 3 shows a comparison of BI-RADS with the synthesised measurement for elastography, and it shows that using BI-RADS diagnosed significantly fewer participants

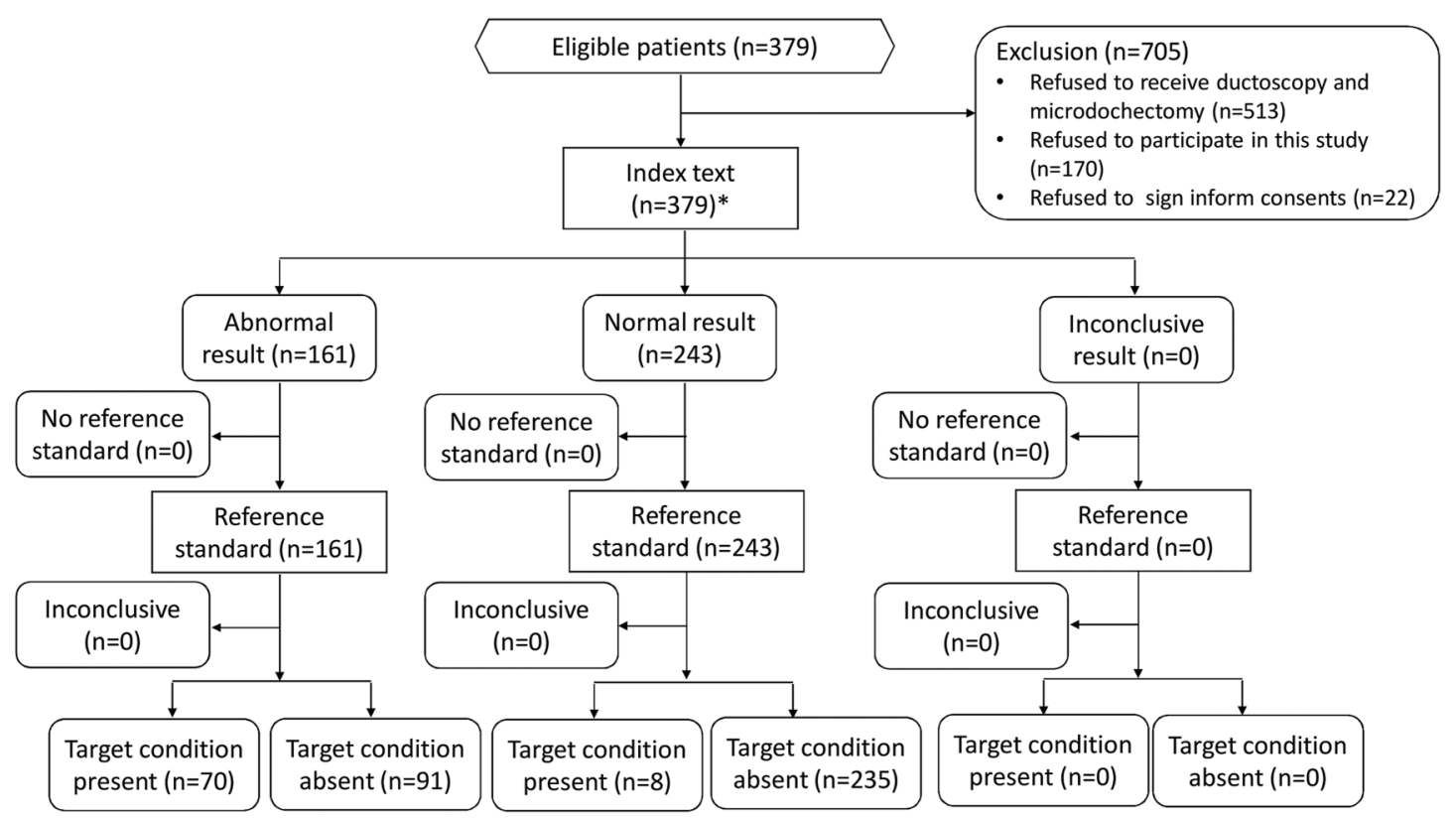

Figure 1 Flow chart of this study. * There were 379 participants presenting 404 lesions. In the results, we counted the number of lesions instead of the patients. The index test is shear wave elastography on the basis of B-mode ultrasound. The reference test is ductoscopy and subsequent microdochectomy if pathological changes were found in ductoscopy. 


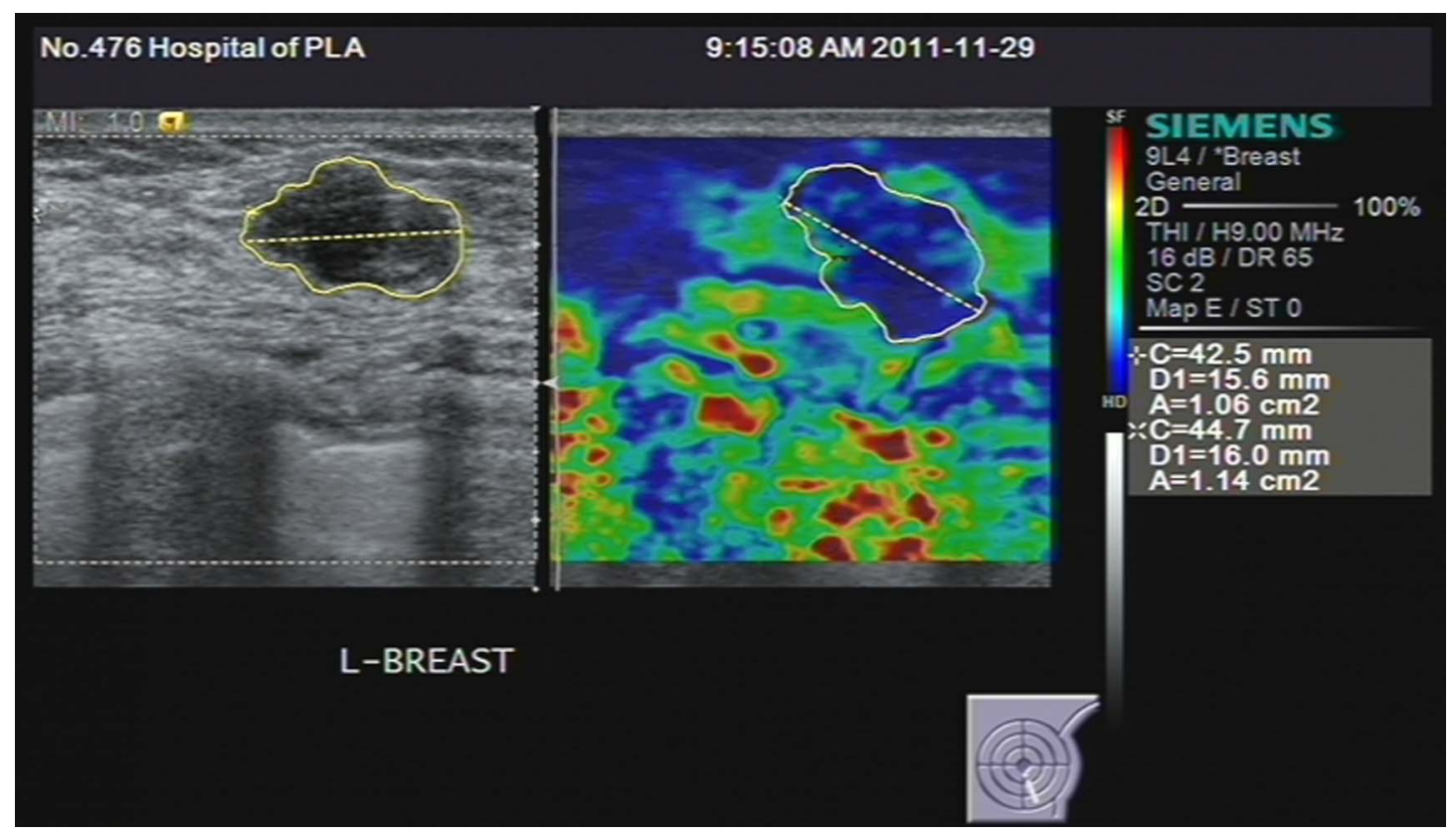

Figure 2 The image of a 45-year-old patient with invasive ductal carcinoma. The left picture showing a B-mode ultrasonography locating the region of interest (ROI). The right picture showing the elastography of the breast lesion, with a blue colour indicating a harder tissue and a red colour indicated a softer tissue.

with malignancy than the synthesised measurement did $\left(\chi^{2}=72.035, \mathrm{p}<0.001\right)$.

The biopsy results showed that $326(80.6 \%)$ breast lesions were classified as benign while 78 (19.4\%) were malignant. We used the McNemar's test to compare the synthesised measurement with the biopsy results, this showed that significantly more malignant lesions were found by elastography with the synthesised measurement (McNemar's $\left.\chi^{2}=67.919, \mathrm{p}<0.001\right)$. The sensitivity, specificity, PPV and NPVs were $0.897,0.721,0.435$ and 0.967 , respectively. Table 2 shows the raw numbers and diagnostic estimates of the synthesised measurement. In the sensitivity analysis, we excluded 152 participants who were found with no pathological changes by ductoscopy and included 227 participants. These participants presented with 237 breast lesions. The measurement of synthesised score categorised $168(70.9 \%)$ breast lesions as benign and $69(29.1 \%)$ as malignant. Results of the reference test showed that $159(67.1 \%)$ of the 237 lesions were benign and the other 78 (32.9\%) were

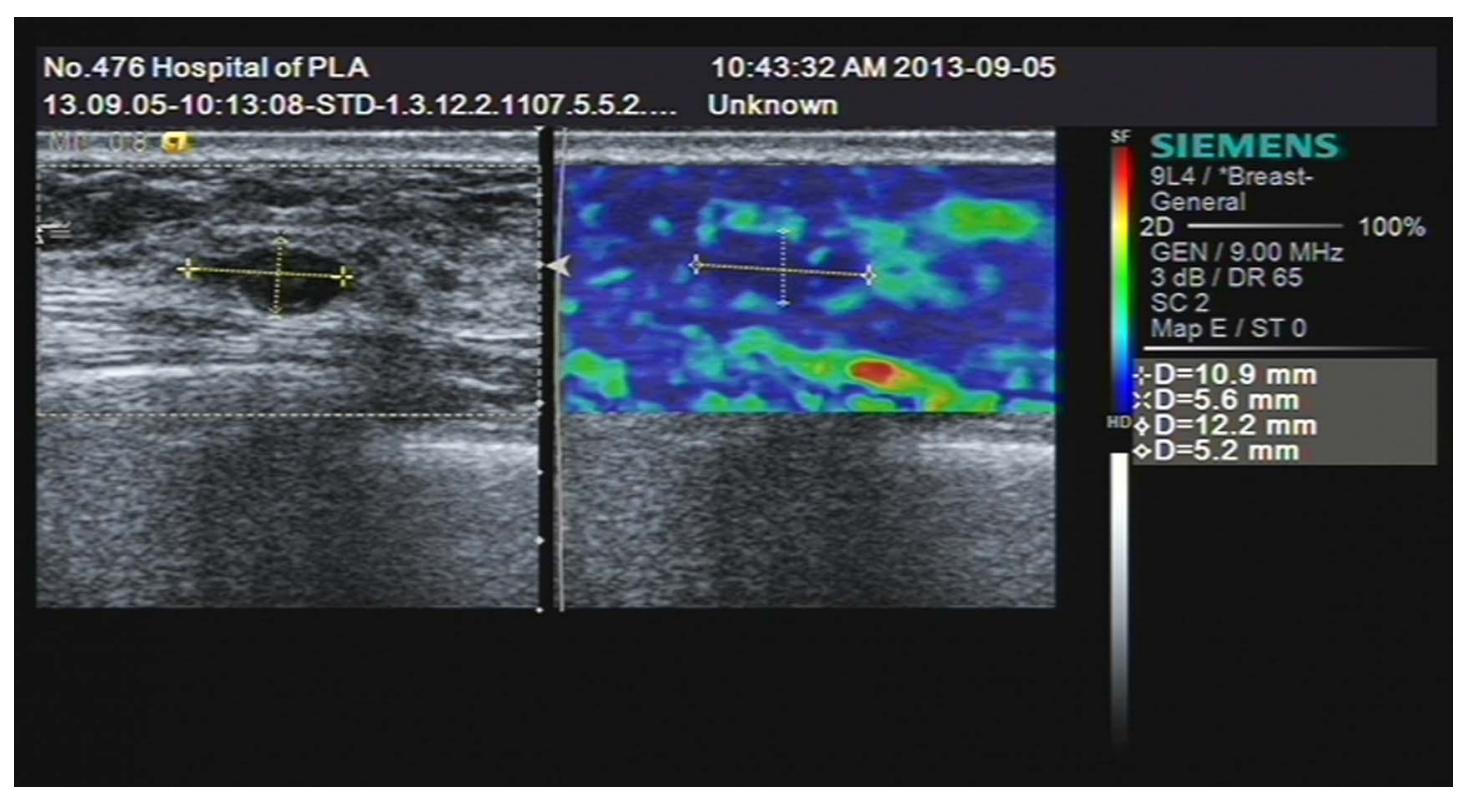

Figure 3 The image of a 41-year-old patient with fibroadenoma. B-mode ultrasonography at the left showing the location of the region of interest $(\mathrm{ROI})$. An elastography picture on the right shows the elasticity of the fibroadenoma. 
Table 2 Predictive value of the synthesised quantitative measurement

\begin{tabular}{|c|c|c|c|c|c|c|}
\hline Measurements & $\begin{array}{l}\text { Correctly } \\
\text { positive }\end{array}$ & $\begin{array}{l}\text { Correctly } \\
\text { negative }\end{array}$ & Sensitivity (\%) & Specificity (\%) & PPV (\%) & NPV (\%) \\
\hline $\begin{array}{l}\text { Synthesised score } \\
(n=404)\end{array}$ & $70 / 78$ & $235 / 326$ & $\begin{array}{l}89.7 \text { ( } 83.3 \text { to } \\
96.2)\end{array}$ & $\begin{array}{l}72.1(66.9 \text { to } \\
77.0)\end{array}$ & $\begin{array}{l}43.5 \text { (35.8 to } \\
51.1)\end{array}$ & $\begin{array}{l}96.7 \text { (94.5 to } \\
99.0)\end{array}$ \\
\hline
\end{tabular}

malignant. The sensitivity and specificity were 0.820 and 0.968 , respectively.

\section{DISCUSSION}

The results of our study showed that a combination of qualitative and quantitative measurements for SWE elastography is sensitive in predicting malignancy in patients with PND, which is consistent with several recent studies testing the diagnostic value of elastography in breast masses. ${ }^{8} 1621$ However, several studies reported that ultrasonography was not predictive of malignancy in patients with PND. ${ }^{22-24}$ We discuss our major findings and answer the remaining questions below.

Predicting malignancy in patients with PND is still challenging, Sabel et al found that $95 \%$ of PND lesions are benign and need not be excised. However, most patients will choose duct excision for palliative purposes. ${ }^{25}$ In the screen phase of our study, 53 patients refused to undergo ductoscopy or duct excision and were therefore excluded. The patients refused to take the examinations as no evidence of malignancy had so far been found and they were afraid of the contraindications of ductoscopy or duct excision. Therefore, a noninvasive method with accurate diagnosis is needed for patients with PND. Alcock and Layer found no predictive value of ductoscopy and ultrasonography in differentiating benign and malignant breast lesions in patients with PND. The authors of this study suggested that ductal surgery was the only reliable way of providing a diagnosis. ${ }^{17}$ In our study, we found that SWE has a sensitivity of $90 \%$ for screening malignancy in patients with PND. This result indicates that SWE elastography could be used as a helpful triage test before advising patients with PND to undergo ductoscopy examinations. Also, compared with BUS, SWE elastography has a lower falsenegative risk, according to the comparison of the BI-RADS and synthesised measurements in our study. Therefore SWE has better diagnostic performance than BUS. Our study, for the first time, revealed that ultrasonographic elastography is valuable in predicting malignant lesions in patients with PND.

The size of the breast mass might be an important factor in differentiating benign and malignant lesions. We found that patients with PND with malignant lesions presented a bigger breast mass (malignant vs benign, $15.58 \mathrm{~mm}$ vs $8.15 \mathrm{~mm}$ ), which might be easier to be detected using BUS and elastography. This finding is consistent with other studies using elastography to detect breast lesions. ${ }^{81}$ However, whether the size of the breast mass could be used as an independent indicator for predicting breast malignancy needs more studies.

It is interesting to find that 513 patients with PND refused to undergo ductoscopy examination in our

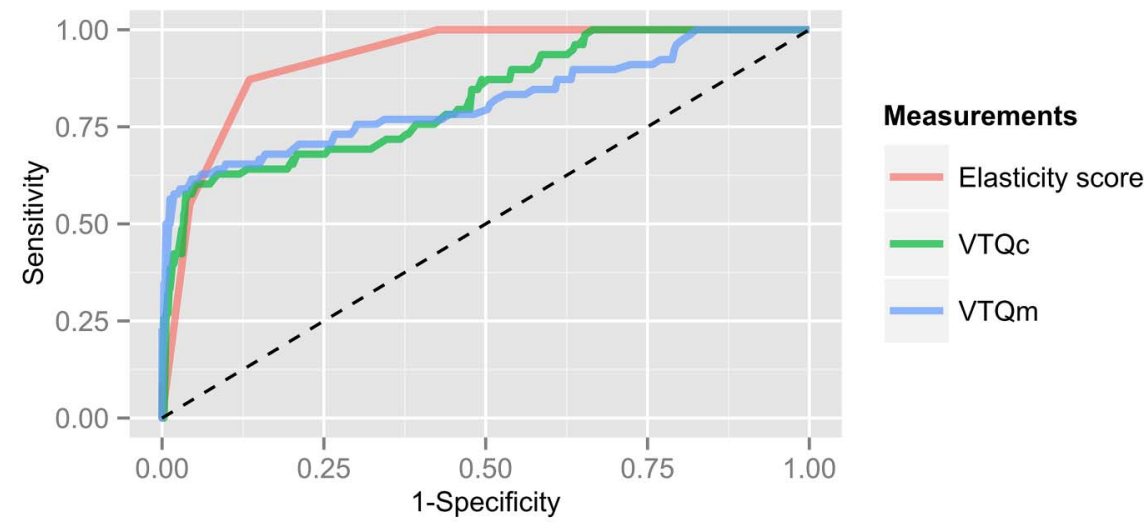

Figure 4 The receiver-operating-characteristics (ROC) curve of elasticity score, VTQm and VTQc. VTQm, virtual touch quantification at margin of a lesion. VTQc, virtual touch quantification at the centre of a lesion. The figure shows the area under the curve (AUC), specificity and sensitivity of Elasticity score, VTQm and VTQc. A cut-off point of 2.50 was selected for elasticity score yielding a sensitivity of $0.872(95 \% \mathrm{Cl} 0.795$ to 0.940$)$, a specificity of $0.736(95 \% \mathrm{Cl} 0.660$ to 0.805$)$ and an AUC of 0.854 . A cut-off point of $2.860 \mathrm{~m} / \mathrm{s}$ was selected for VTQm yielding a sensitivity of 0.731 ( $95 \% \mathrm{Cl} 0.628$ to 0.821 ), a specificity of 0.742 $(95 \% \mathrm{Cl} 0.673$ to 0.805$)$ and an AUC of 0.825 . Additionally, a cut-off point of $3.015 \mathrm{~m} / \mathrm{s}$ was calculated for VTQc with a sensitivity of $0.731(95 \% \mathrm{Cl} 0.628$ to 0.821$)$, a specificity of $0.698(95 \% \mathrm{Cl} 0.616$ to 0.761$)$ and an AUC of 0.857 . 
Table 3 The BI-RADS classification and the synthesised measures

\begin{tabular}{llllll}
\hline BI-RADS* & $\begin{array}{l}\text { Elasticity score } \\
\text { Mean (SD) }\end{array}$ & $\begin{array}{l}\text { VTQm } \\
\text { Mean (SD) }\end{array}$ & $\begin{array}{l}\text { VTQc } \\
\text { Mean (SD) }\end{array}$ & \multicolumn{2}{l}{ Synthesised score } \\
\hline $3(n=293)$ & $1.98(0.64)$ & $2.64(0.51)$ & $2.86(0.55)$ & $207(85.2)$ & $86(53.4)$ \\
4A $(n=46)$ & $2.84(0.68)$ & $2.85(0.76)$ & $3.02(0.76)$ & $25(10.3)$ & $21(13.1)$ \\
4B $(n=23)$ & $3.91(0.29)$ & $3.24(0.86)$ & $3.48(0.78)$ & $4(1.6)$ & $19(11.8)$ \\
$4 C(n=16)$ & $3.71(0.61)$ & $2.90(0.70)$ & $3.34(0.84)$ & $5(2.1)$ & $11(6.8)$ \\
$5(n=26)$ & $4.00(0.81)$ & $3.31(0.62)$ & $3.71(0.75)$ & $2(0.8)$ & $24(14.9)$ \\
\hline
\end{tabular}

BI-RADS, the Breast Imaging Reporting and Data System; N, number of counts; VTQc, virtual touch quantification at the centre of a lesion; VTQm, virtual touch quantification at the margin of a lesion.

*In the BI-RADS, number 3 refers to probably benign, number 4 refers to suspicious malignancy and number 5 refers to highly suggestive of malignancy. In the 4 category, a subclassification is used: (1) 4A: low suspicious for malignancy; (2) 4B: intermediate suspicious for malignancy; (3) 4C: moderate concern, but not classic for malignancy.

study. Absence of these patients might have contributed to a high incidence of malignant breast lesions, and thus overestimated the diagnostic value of elastography. The reason for this may be that patients with PND worried about postoperative pain and procedure cost, so they turned to other non-invasive tests. When the noninvasive tests showed no positive findings, they refused to take ductoscopy. This is the reason we carried out this study - to find a reliable and pain-free test for individuals such as these.

The synthesised score we used in this study is similar to a published method for classification of benign and malignant breast lesions, except that we did not include the BI-RADS. ${ }^{8}$ The BI-RADS is a classification method that is in part coincidental with the elasticity score. ${ }^{19}$ We chose elasticity score instead of the BI-RADS in the synthesised score. The elasticity score is a qualitative measurement; it has been treated as continuous data in several studies. ${ }^{82126}$ We also treated the elasticity score as continuous data, so that we could calculate a cut-off point and include it into the synthesised score. The way we handled the elasticity score may bring bias into our results. However, we did not find a better way to solve the problem, and neither did the previous studies. Therefore, in future studies, a qualitative measurement should be developed that can differentiate benign and malignant breast lesions more clearly than the elasticity score.

In this study, we incorporated VTQ both at the margin (VTQm) and in the centre (VTQc) into the synthesised score. To the best of our knowledge, we are the first to use these two quantitative measurements; previous studies used VTQc alone. ${ }^{8} 921$ Inclusion of both VTQm and VTQc could increase the understanding of breast lesions, since they were reported to be different. ${ }^{19} 21$ The results of our study indicated that both VTQm and VTQc were significantly higher in malignant than in benign lesions. We might have assigned more weight to the VTQ measurement, and therefore made breast lesions with higher VTQ easier to be malignant in the synthesised score.

There are several limitations in this study. First, a total of 152 participants were found with no pathological change after ductoscopy examination, and thus microdochectomy to get a biopsy result was not performed.
These participants were classified as having benign lesions, without solid evidence. We might have missed the malignant lesions, since ductoscopy could only reach a depth of $2 \mathrm{~cm} .{ }^{5}$ To ensure the reliability of our study, we ran a sensitivity analysis, in which all these participants were classified as with malignant lesions. And we found the result was consistent. Moreover, we found that the incidence of malignancy in patients with PND is $19.3 \%$ in our study, while the reported incidence ranges from $4 \%$ to $29 \% .{ }^{27-30}$ This supported the classification of 152 participants as having benign lesions. Second, the weight of the elasticity score, VTQm and VTQc, were assigned evenly, and therefore made the value of VTQ a more important factor than elasticity score in predicting malignancy in patients with PND. Third, the surgeon who performed the ductoscopy was not blinded from the ultrasonographic results, which may introduce performance bias.

In conclusion, ultrasonography elastography is sensitive for patients with PND and could be considered as a triage test before ductoscopy examination. However, a study for further improvement of diagnostic sensitivity is warranted by assigning proper weights to the qualitative and quantitative measurements for elastography.

Acknowledgements This study was financially supported by the Key Project of Science and Technology of Fujian Province of China (number 2009Y0043). The authors would like to thank all the participants who took part in this study.

Contributors $X G$ and $Y L$ contributed to the design and manuscript writing. $X G$ and $W L$ conceptualised the study and data analysis. $Y L$ assisted with data collection. All the authors read and approved the final manuscript.

Funding This research received no specific grant from any funding agency in the public, commercial or not-for-profit sectors.

Competing interests None declared.

Patient consent Obtained.

Ethics approval The ethical review board of Fuzhou General Hospital of Nanjing Military Command provided ethical approval (number FZJQ2011018).

Provenance and peer review Not commissioned; externally peer reviewed.

Data sharing statement No additional data are available.

Open Access This is an Open Access article distributed in accordance with the Creative Commons Attribution Non Commercial (CC BY-NC 4.0) license, which permits others to distribute, remix, adapt, build upon this work non- 
commercially, and license their derivative works on different terms, provided the original work is properly cited and the use is non-commercial. See: http:// creativecommons.org/licenses/by-nc/4.0/

\section{REFERENCES}

1. Siegel R, Ma J, Zou Z, et al. Cancer statistics, 2014. CA Cancer J Clin 2014;64:9-29.

2. Fan L, Strasser-Weippl K, Li JJ, et al. Breast cancer in China. Lancet Oncol 2014;15:e279-89.

3. Chen L, Zhou WB, Zhao Y, et al. Bloody nipple discharge is a predictor of breast cancer risk: a meta-analysis. Breast Cancer Res Treat 2012;132:9-14.

4. Jagmohan P, Pool FJ, Putti TC, et al. Papillary lesions of the breast: imaging findings and diagnostic challenges. Diagn Interv Radiol 2013;19:471-8.

5. Denewer A, El-Etribi K, Nada N, et al. The role and limitations of mammary ductoscope in management of pathologic nipple discharge. Breast J 2008;14:442-9.

6. Makita M, Akiyama F, Gomi N, et al. Mammary ductoscopy and watchful follow-up substitute microdochectomy in patients with bloody nipple discharge. Breast cancer 2014:1-10.

7. Kooistra BW, Wauters C, van de Ven S, et al. The diagnostic value of nipple discharge cytology in 618 consecutive patients. Eur J Surg Oncol 2009;35:573-7.

8. Kim YS, Park JG, Kim BS, et al. Diagnostic value of elastography using acoustic radiation force impulse imaging and strain ratio for breast tumors. J Breast Cancer 2014;17:76-82.

9. Tozaki M, Isobe S, Sakamoto M. Combination of elastography and tissue quantification using the acoustic radiation force impulse (ARFI) technology for differential diagnosis of breast masses. Jpn J Radiol 2012;30:659-70.

10. Tozaki M, Isobe S, Fukuma E. Preliminary study of ultrasonographic tissue quantification of the breast using the acoustic radiation force impulse (ARFI) technology. Eur J Radiol 2011;80:e182-7.

11. Meng W, Zhang G, Wu C, et al. Preliminary results of acoustic radiation force impulse (ARFI) ultrasound imaging of breast lesions. Ultrasound Med Biol 2011;37:1436-43.

12. Taylor K, O'Keeffe S, Britton PD, et al. Ultrasound elastography as an adjuvant to conventional ultrasound in the preoperative assessment of axillary lymph nodes in suspected breast cancer: a pilot study. Clin Radiol 2011;66:1064-71.

13. Sadigh $\mathrm{G}$, Carlos $\mathrm{RC}, \mathrm{Neal} \mathrm{CH}$, et al. Accuracy of quantitative ultrasound elastography for differentiation of malignant and benign breast abnormalities: a meta-analysis. Breast Cancer Res Treat 2012;134:923-31.

14. Gong $X, X u Q, X u Z$, et al. Real-time elastography for the differentiation of benign and malignant breast lesions: a meta-analysis. Breast Cancer Res Treat 2011;130:11-18.
15. Kılıç F, Ustabaşıoğlu FE, Samancı C, et al. Ductal carcinoma in situ detected by shear wave elastography within a fibroadenoma. $J$ Breast Cancer 2014;17:180-3.

16. Youk JH, Son EJ, Gweon HM, et al. Comparison of strain and shear wave elastography for the differentiation of benign from malignant breast lesions, combined with B-mode ultrasonography: qualitative and quantitative assessments. Ultrasound Med Biol 2014;40:2336-44.

17. Alcock C, Layer GT. Predicting occult malignancy in nipple discharge. ANZ J Surg 2010;80:646-9.

18. Sanders MA, Roland L, Sahoo S. Clinical implications of subcategorizing BI-RADS 4 breast lesions associated with microcalcification: a radiology-pathology correlation study. Breast $J$ 2010;16:28-31

19. Itoh A, Ueno E, Tohno E, et al. Breast disease: clinical application of US elastography for diagnosis. Radiology 2006;239:341-50.

20. Wu S. Medical statistics. BeiJing: People's Medical Publishing House, 2002:164-78.

21. Li G, Li DW, Fang YX, et al. Performance of shear wave elastography for differentiation of benign and malignant solid breast masses. PLOS ONE 2013;8:e76322.

22. Munoz Carrasco R, Alvarez Benito M, Rivin del Campo E. Value of mammography and breast ultrasound in male patients with nipple discharge. Eur J Radiol 2013;82:478-84.

23. Hamed ST, Abdo MH, Ahmed HH. Breast discharge: ultrasound and Doppler evaluation. J Egypt Natl Canc Inst 2008;20:262-70.

24. Parajuly SS, Lan PY, Yun MB, et al. Diagnostic potential of strain ratio measurement and a 5 point scoring method for detection of breast cancer: Chinese experience. Asian Pac J Cancer Prev 2012;13:1447-52.

25. Sabel MS, Helvie MA, Breslin T, et al. Is duct excision still necessary for all cases of suspicious nipple discharge? Breast $J$ 2012;18:157-62.

26. Choi JJ, Kang BJ, Kim SH, et al. Role of sonographic elastography in the differential diagnosis of papillary lesions in breast. Jpn J Radiol 2012;30:422-9.

27. Seltzer MH. Breast complaints, biopsies, and cancer correlated with age in 10,000 consecutive new surgical referrals. Breast $J$ 2004:10:111-17.

28. Lau S, Kuchenmeister I, Stachs A, et al. Pathologic nipple discharge: surgery is imperative in postmenopausal women. Ann Surg Oncol 2005;12:546-51.

29. Vargas HI, Vargas MP, Eldrageely K, et al. Outcomes of clinical and surgical assessment of women with pathological nipple discharge. Am Surg 2006;72:124-8.

30. Morrogh M, Morris EA, Liberman L, et al. The predictive value of ductography and magnetic resonance imaging in the management of nipple discharge. Ann Surg Oncol 2007;14:3369-77. 